\title{
Comparação clínica de dois sistemas digitais de radiografias intraorais
}

\author{
A comparison between two digital systems of intraoral radiographs \\ Comparación clínica de dos sistemas digitales de radiografías intraorales \\ Carla Renata Sanomiya IKUTA ${ }^{1}$ \\ Leda Maria Pescinini SALZEDAS ${ }^{1}$ \\ Departamento de Patologia e Propedêutica Clínica, Disciplina de Radiologia Odontológica, \\ Faculdade de Odontologia de Araçatuba, UNESP-Univ. Estadual Paulista, 16015-050 Araçatuba-SP, Brasil
}

\begin{abstract}
Resumo
Introdução: $O$ avanço da tecnologia permitiu que a Radiologia Odontológica evoluísse para o sistema digital, eliminando o processamento químico das radiografias e otimizando o tempo clínico. Todavia, o clínico necessita manipular cada sistema digital, considerando as similaridades e diferenças para o melhor uso em clínica. Objetivo: Discutir as técnicas dos dois tipos de sensores digitais disponíveis no Setor de Radiologia da FOA-UNESP. Material e Método: Os dois sistemas de imagem digital, placa de fósforo fotoestimulável (PSP) e sensor sólido (CMOS), foram comparados para observação de suas similaridades desde o momento do posicionamento no paciente, processamento da imagem até o armazenamento, e também considerando o custo da implantação de cada sistema. Resultados: Os posicionadores para o sistema CMOS são diferentes dos utilizados nas radiografias convencionais. O processamento da imagem das placas de fósforo necessita de um aparelho de escaneamento adicional de alto custo, e é passível de erro durante a manipulação. Já o sistema CMOS, tem a imagem adquirida imediata e concomitantemente ao disparo do aparelho de raios X, mas necessita de maior cuidado para a inserção do conjunto posicionador-sensor no paciente. Conclusão: A placa de fósforo representa o sistema de implantação de maior custo, mas é o sistema mais confortável para o paciente e mais semelhante, em relação ao posicionamento, às radiografias com filme. O sistema CMOS é mais barato e a visualização da imagem é imediata. Ambos permitem que a imagem seja editada e armazenada em computadores.
\end{abstract}

Descritores: Radiografia; Radiografia Dentária Digital; Radiografia Dentária.

\section{Abstract}

Introduction: The technology allowed Dental Radiology to evolve into the digital system, eliminating the chemical processing of radiographs and optimizing clinical time. However, the clinician needs to manipulate each digital system, in order to consider the similarities and the differences for the best clinical use. Objective: To discuss the techniques of the two types of digital sensors available in the Radiology Department of FOA-UNESP. Material and Method: The two systems of digital image, photosensitive phosphor plate (PSP) and solid sensor (CMOS), were compared to observe their similarities from the time of patient positionin unitl the image processing to storage, and also considering the cost of deploying each system. Results: The positioners for the CMOS system are different from those used in conventional radiographs. The image processing of the phosphor plates requires an additional expensive scanning device and is susceptible to error during manipulation. On the other hand, the CMOS system has the image acquired immediately and concomitantly with the X-ray device firing, but more care is needed to insert the positioner-sensor assembly into the patient. Conclusion: The phosphor plate represents the costliest implantation system, but it is the most comfortable system for the patient and more similar, in relation to the positioning, to the film X-rays. The CMOS system is cheaper, and the image preview is immediate. Both allow the image to be edited and stored on computers.

Descriptors: Radiography; Radiography, Dental, Digital; Radiography, Dental.

\section{Resumen}

Introducción: El avance de la tecnología permitió que la Radiología Odontológica evolucionara hacia el sistema digital, eliminando el procesamiento químico de las radiografías y optimizando el tiempo clínico. Sin embargo, el clínico necesita manipular cada sistema digital, considerando las similitudes y diferencias para el mejor uso en clínica. Objetivo: Discutir las técnicas de los dos tipos de sensores digitales disponibles en el Sector de Radiología de la FOA-UNESP. Material y Método: Los dos sistemas de imagen digital, placa de fósforo fotoeléctrico (PSP) y sensor sólido (CMOS), fueron comparados para observar sus semejanzas desde el momento del posicionamiento en el paciente, procesamiento de la imagen hasta el almacenamiento, y también considerando el costo de la implementación de cada sistema. Resultados: Los posicionadores para el sistema CMOS son diferentes de los utilizados en las radiografías convencionales. El procesamiento de la imagen de las placas de fósforo necesita un aparato de escaneado adicional de alto costo, y es susceptible de error durante la manipulación. El sistema CMOS, tiene la imagen adquirida inmediata y concomitantemente al disparo del aparato de rayos X, pero necesita de mayor cuidado para la inserción del conjunto posicionadorsensor en el paciente. Conclusión: La placa de fósforo representa el sistema de implantación de mayor costo, pero es el sistema más cómodo para el paciente y más semejante, en relación al posicionamiento, a las radiografías con película. El sistema CMOS es más barato y la visualización de la imagen es inmediata. Ambos, permiten que la imagen sea editada y almacenada en ordenadores.

Descriptores: Radiografía; Radiografía Dental Digital; Radiografía Dental.

\section{INTRODUÇÃO}

As radiografias intraorais analógicas são obtidas por meio de etapas que consistem na aquisição de imagem por meio de um filme que deve ser processado em soluções químicas para a visualização da imagem final. Atualmente, as imagens radiográficas intraorais evoluíram para o sistema digital. As principais vantagens do uso dos sistemas digitais na radiologia são: diminuição de até $90 \%$ da dose de radiação; eliminação do filme radiográfico e do processamento químico; rápido processamento; visualização rápida da radiografia final; reconstrução, edição e compartilhamento das imagens; e controle de exposição automático com alcance dinâmico o que diminui os erros de sub e super-exposição ${ }^{1,2,3}$. Além de serem métodos não poluentes e ecologicamente corretos no que diz respeito aos resíduos químicos gerados pelas soluções reveladoras ${ }^{3}$. Já as desvantagens incluem aspectos como o custo de implementação dos sistemas ${ }^{2}$ e treinamento para adequado para o uso dos sistemas.

As tecnologias presentes no mercado para as radiografias intra-orais são a CMOS (Complementary Metal
Oxide Semiconductor) e PSP (Photostimulable Phosphor Plate $)^{3,4,5}$ conhecidas respectivamente como sensor a cabo e placa de fósforo. Essas tecnologias são classificadas em indiretas e diretas: no primeiro sistema, os fótons de raios $\mathrm{X}$ são primeiramente convertidos em fótons de luz, e posteriormente em sinais elétricos; enquanto que no segundo a conversão dos fótons de raios X para carga elétrica ocorre diretamente $^{3}$. Com base no exposto, o propósito deste trabalho foi comparar as diferenças e similaridades na prática clínica dos dois sistemas disponíveis no setor de Radiologia da Faculdade de Odontologia de Araçatuba (FOA-UNESP)

\section{MATERIAL E MÉTODO}

Os sistemas de imagem por meio do CMOS e PSP foram comparados na execução da técnica, quanto ao uso do posicionador, processamento da imagem e visualização da imagem final, simulando aquisições de radiografias periapicais. Nenhum paciente foi submetido à exposição da radiação em razão do presente trabalho, sendo utilizados os 
seguintes materiais: embalagem de PVC, sistema de CMOS (MicroImagem ${ }^{\circledR}$ ), $\quad$ sistema PSP (Instrumentarium ${ }^{\circledR}$ ), posicionador do tipo Hanshin, posicionador para o sensor tipo CMOS e estação de trabalho computadorizado.

\section{RESULTADOS}

\section{- Sensor e posicionamento}

Já no primeiro manuseio dos sistemas é possível perceber a diferença no receptor da imagem. O dispositivo CMOS é mais espesso e em seu corpo estão acoplados eletrodos e cabo com terminal USB. A PSP tem as dimensões semelhantes ao de um filme intraoral convencional. Devido a essas diferenças, os posicionadores utilizados para cada tecnologia são distintos: a PSP utiliza o posicionador convencional do tipo Hanshin, e o CMOS, o posicionador oferecido pelo fabricante (FiguraS 1 e 2). Assim como em um filme radiográfico intraoral convencional, ambos os sistemas apresentam uma face de exposição, análoga à face lisa de um filme convencional que deve estar voltada para o feixe útil de Raios-X no momento do disparo.

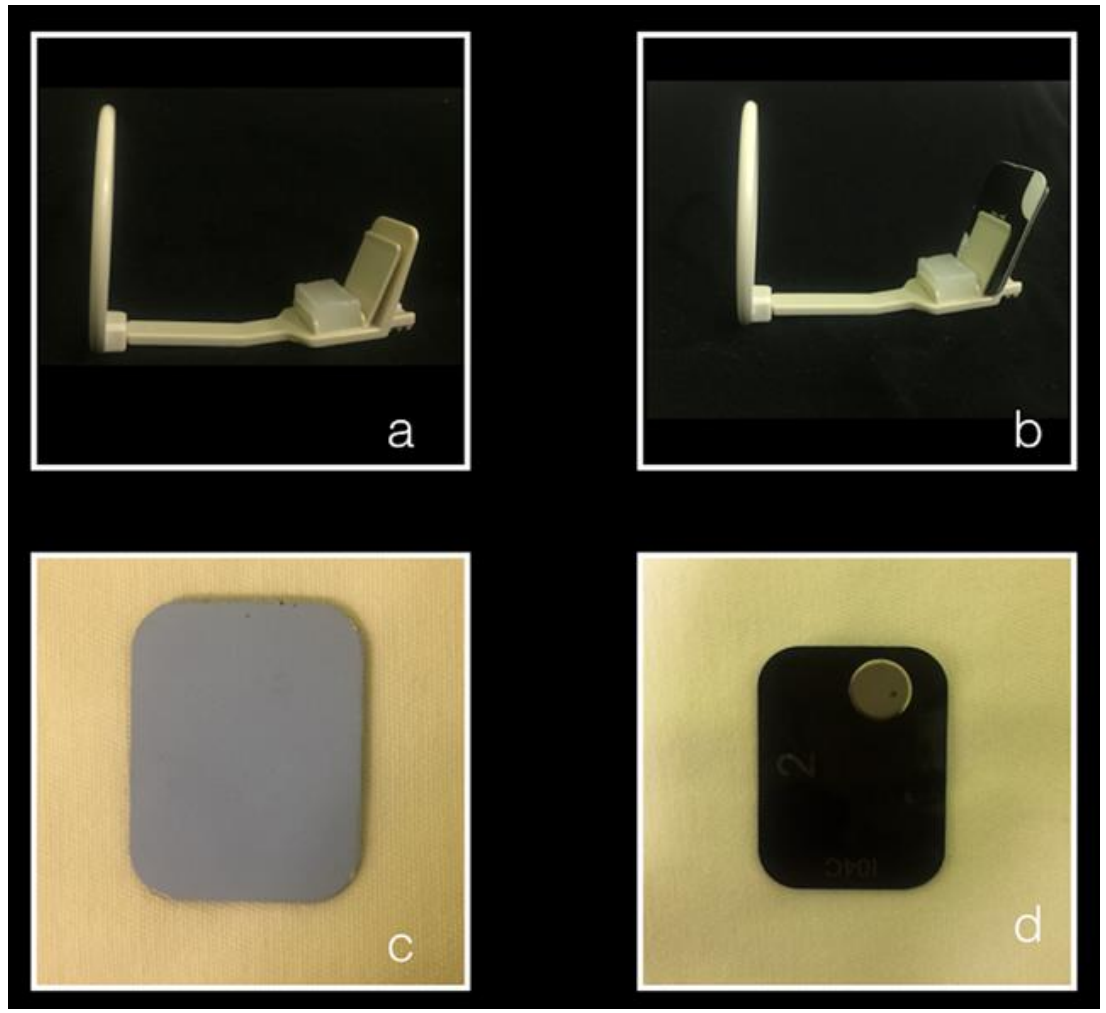

Figura 1: Posicionador do tipo Hanshin(a); Sensor de placa de fósforo fotoestimulável colocado no posicionador, protegido por capa de papelão fornecida pelo fabricante (b); Face de exposição à radiação $\mathrm{X}$ do sensor de placa da fósforo fotoestimulável (c) e face contrária a exposição à radiação, que contém um imã para auxílio no escaneamento da radiografia (d).
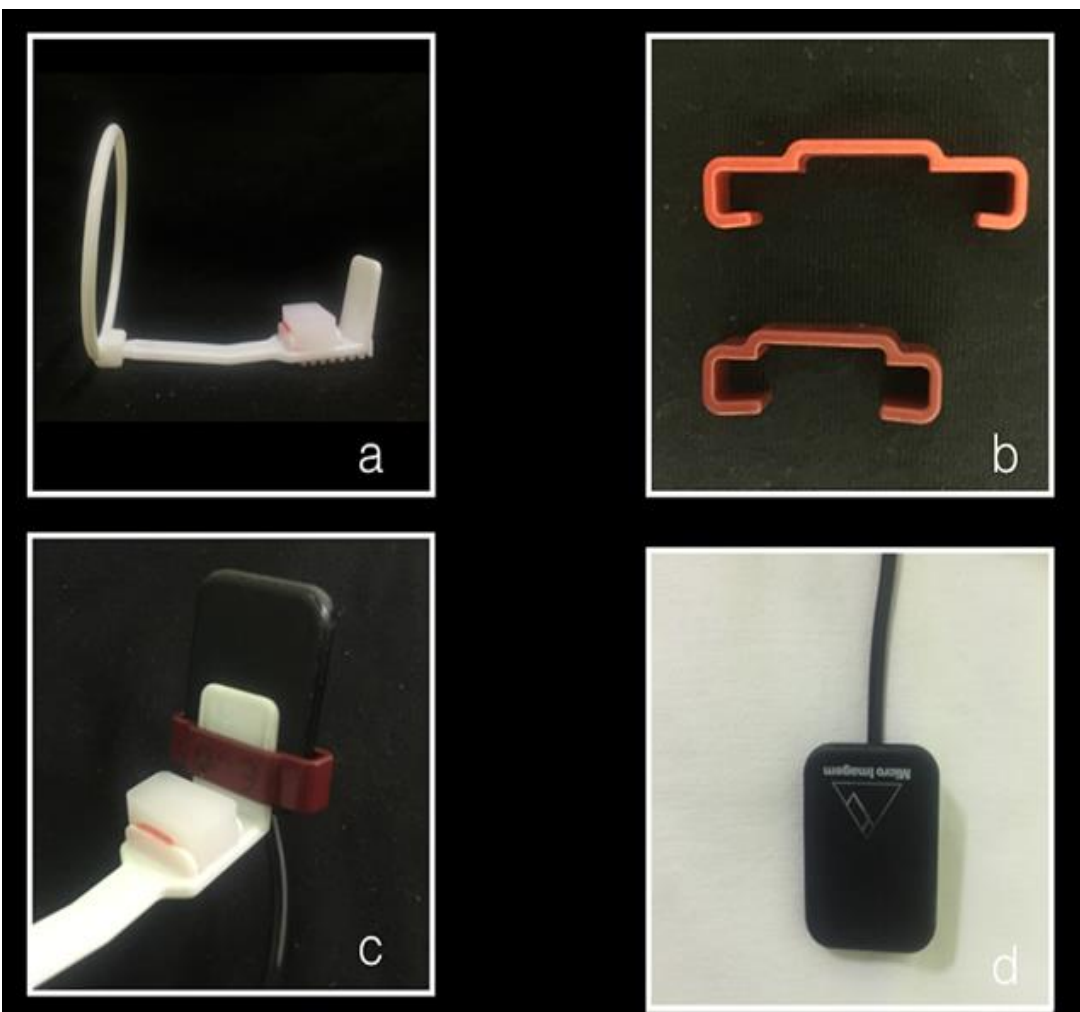

Figura 2: Posicionador específico para o sensor CMOS (a); Presilhas que auxiliam na colocação do sensor ao posicionador, sendo a presilha menor para as radiografias de dentes anteriores e a maior para os posteriores (b); Parte com elevação na presilha, voltada para a face lisa do sensor, formando um sistema de encaixe para que o sensor fique estabilizado no posicionador (c); Face de exposição do sensor CMOS (d).

\section{○ Processamento da imagem}

Ambos os sistemas necessitam de uma estação de trabalho computadorizada. O processamento da imagem é praticamente imediato no sensor do tipo CMOS, ou seja, a imagem é produzida quase concomitantemente ao disparo do aparelho de Raios X. Já no sistema do tipo PSP, a placa deve ser escaneada para que a imagem latente seja visualizada no monitor, e, portanto, o processamento da imagem é operador-dependente (Figura 3).

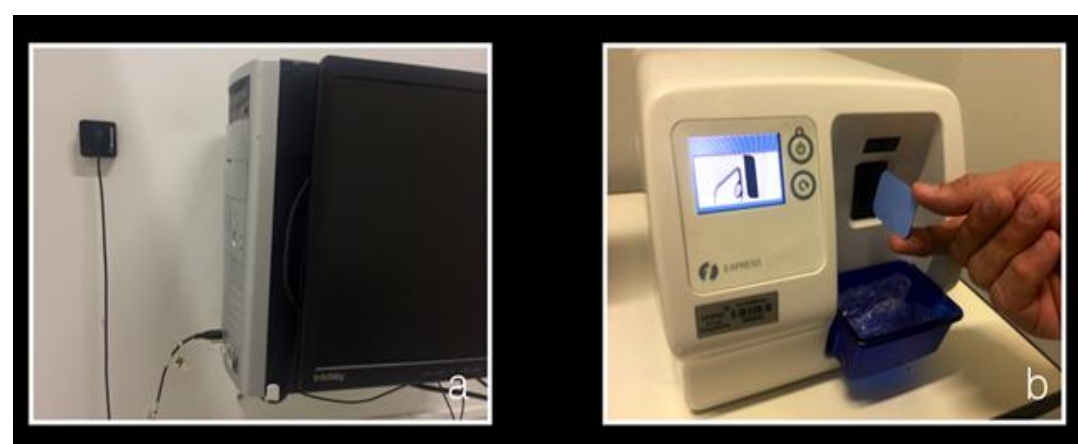

Figura 3: Sensor CMOS acoplado a saída USB do computador, que possibilita que a imagem seja visualizada imediatamente ao disparo do aparelho de raios X (a); Posicionamento do sensor de placa de fósforo fotoestimulável no escaner para leitura da radiografia final (b).

\section{- Imagem final}

Uma vez adquirida e visualizada a imagem pode ser armazenada em formatos digitais de imagem e ser editada de acordo com a necessidade clínica do cirurgião-dentista. A imagem final do CMOS apresenta uma sombra radiopaca generalizada e menor área de imagem em comparação com a radiografia analógica e PSP (Figura 4).

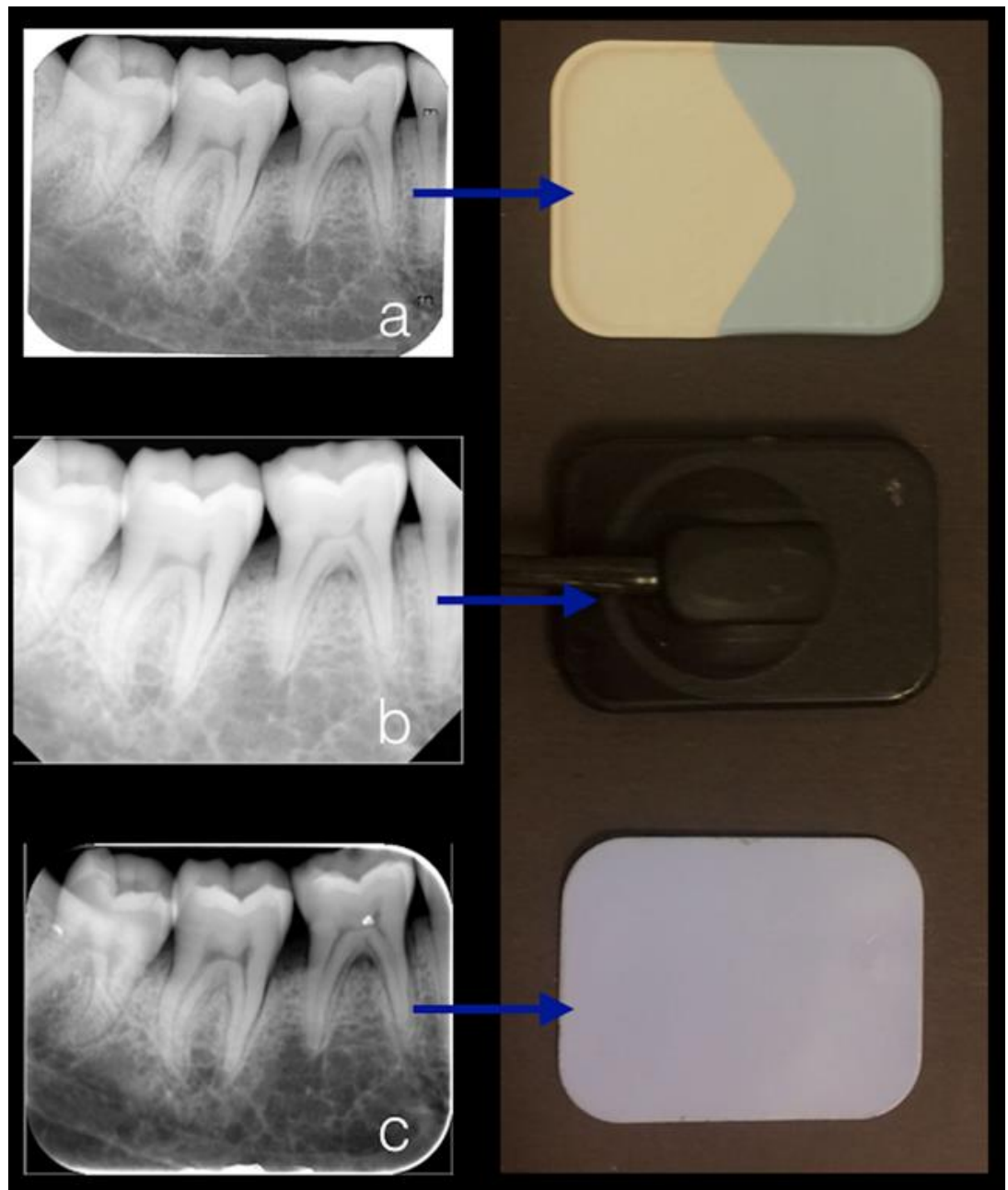

Figura 4: Comparação da imagem final da radiografia entre os três sistemas: analógico (a), CMOS(b) e placa de fósforo fotoestimulável (c)

As principais similaridades e diferenças estão descritas nos Quadros 1 e 2

\section{Quadro 1: Similaridades entre os sistemas PSP e CMOS}

Inserção do sensor no posicionador : face lisa voltada para o feixe de Raios-X Edição de imagem: manipulação do brilho, contraste, saturação, mensuração de estruturas e outros recursos úteis para o diagnóstico

Armazenamento: em rede ou HD externo, nos formatos de imagem digita Biossegurança: embalagem de PVC 
Quadro 2: Diferenças entre os sistemas PSP e CMOS

\begin{tabular}{|l|l|l|}
\hline $\begin{array}{c}\text { Etapa/ } \\
\text { procedimento } \\
\text { clínico }\end{array}$ & \multicolumn{1}{|c|}{ PSP } & \multicolumn{1}{|c|}{ CMOS } \\
$\begin{array}{l}\text { Posicionador } \\
\text { Posicionador } \\
\text { convencional } \\
\text { (tipo Hanshin) }\end{array}$ & $\begin{array}{l}\text { Específico e com presilhas } \\
\text { para a apreensão do sensor ao } \\
\text { posicionador }\end{array}$ \\
$\begin{array}{l}\text { Sensor enão do } \\
\text { posicionador no } \\
\text { paciente }\end{array}$ & $\begin{array}{l}\text { Similar ao } \\
\text { posicionamento } \\
\text { com filme } \\
\text { analógico }\end{array}$ & $\begin{array}{l}\text { Maior grau de dificuldade para } \\
\text { inserção e maior desconforto } \\
\text { ao paciente O operador deve } \\
\text { atentar-se ao fio do sensor } \\
\text { para evitar que seja rompido } \\
\text { pela mordida do paciente } \\
\text { durante o procedimento }\end{array}$ \\
\hline $\begin{array}{l}\text { Processamento e } \\
\text { visualização da } \\
\text { imagem }\end{array}$ & $\begin{array}{l}\text { A placa deve ser } \\
\text { escaneada para a } \\
\text { visualização da } \\
\text { imagem latente }\end{array}$ & $\begin{array}{l}\text { Quase imediatamente após o } \\
\text { disparo }\end{array}$ \\
\hline $\begin{array}{l}\text { Imagem final } \\
\text { Densidade e área } \\
\text { semelhante às } \\
\text { radiografias } \\
\text { analógicas }\end{array}$ & $\begin{array}{l}\text { Sombra radiopaca e área de } \\
\text { imagem menor em } \\
\text { comparação com as } \\
\text { radiografias convencionais e } \\
\text { PSP }\end{array}$ \\
\hline
\end{tabular}

\section{DISCUSSÃO}

O primeiro sistema digital de radiografia intraoral foi o RadioVisioGraphy (Trophy Radiologie, Vincennes, França), lançado em 1985 e utilizava a tecnologia de CCD (Charge Coupled Device), que antecedeu a tecnologia do $\mathrm{CMOS}^{1}$. Posteriormente, em 1994, a PSP foi comercializada pelo sistema Digora ${ }^{\circledR}$ (Soredex, Helsinque, Finlândia), com a finalidade de diminuir as dificuldades operacionais do sistema $\mathrm{CCD}^{1,2}$. O sistema Digora ${ }^{\circledR}$ utiliza de uma placa óptica digital para obtenção da imagem em um processo denominado de estimulação fósforo-luminescente ${ }^{2}$. As imagens digitais das radiografias são classificadas em indiretas, semi-diretas e diretas $^{1}$. As imagens indiretas são basicamente as radiografias obtidas em filme convencional e depois digitalizadas em escâner, por exemplo ${ }^{1}$.

As PSP por sua vez, estão classificadas nos tipos de imagem semi-direta, também conhecidos como radiologia computadorizada ${ }^{1,3}$. Nesse tipo de sistema a aquisição de imagem, processamento e armazenamento são realizadas de maneira independente ${ }^{1}$. O sistema é composto por uma estação de trabalho com computadores e programas, unidade leitora e as placas de fósforo ${ }^{3}$. Quando a placa é exposta a radiação $X$, um elétron do íon európio é excitado e preso na estrutura cristalina de armazenamento da placa, causando luminescência ${ }^{1}$. Os compostos químicos da placa de fósforo devem ser estimulados pela luz do laser para que a energia armazenada na imagem latente seja liberada ${ }^{1,3}$. Durante a varredura a laser, a quantidade de energia armazenada é liberada em forma de luz e é diferente em cada parte da placa $^{1,2,3}$. Essa luz fluorescente tem energia proporcional a dose de radiação absorvida, liberada e convertida em um sinal elétrico amplificado ${ }^{1}$. Então, o sinal analógico é convertido para um formato digital por meio de um conversor analógicodigital, resultando na radiografia digital final ${ }^{1,3}$

As imagens digitais radiográficas do tipo direta são as adquiridas por meio de sensores sólidos, os CCD e CMOS. Esse tipo de sistema é classificado como direto porque o feixe de raios $\mathrm{X}$ é convertido diretamente em carga elétrica, para que seja formada então a imagem final ${ }^{3}$. Há um fio rígido que conecta o sensor ao computador, que inicia automaticamente a aquisição da imagem quando o nível de radiação é aumentado, dessa maneira, a imagem é formada no monitor em poucos segundos ${ }^{1}$. Os dispositivos sólidos nada mais são do que aglomerados de células fotoelétricas, que são quebradas durante a exposição aos raios $\mathrm{X}$ e geram voltagem na proporção da quantidade de emissão de energia. A quantidade de energia necessária para a quebra de uma célula é de aproximadamente $1,1 \mathrm{~V}$. A carga é lida por transferência de coleta de carga em cada pixel, e depois é destruída, o que resulta em uma imagem latente elétrica que é distribuída em um padrão de cargas na matriz da imagem. Essa informação radiográfica analógica é convertida em um sinal digital, o qual é armazenado em um computador ${ }^{1}$. A principal vantagem desse tipo de sistema é que economiza tempo no processamento da imagem ${ }^{3}$.

As radiografias digitais (RD) representam um avanço no diagnóstico, pois geram uma análise quantitativa na visualização das imagens que até o presente momento eram qualitativos, além de compensar a limitação visual humana por meio da alteração de brilho e contraste ${ }^{4}$. A qualidade da RD está associada ao número de bits por pixel, tamanho do pixel e matriz do equipamento ${ }^{3}$. A matriz é responsável por localizar e quantificar os tons de cinza referente a estrutura, determinando os diferentes contrastes ${ }^{3}$.

Para imagem digital ser considerada de boa qualidade devem ser consideradas as seguintes unidades: densidade ótica, detalhe, contraste e densidade ${ }^{3}$. A densidade ótica é a propriedade que descreve o grau de enegrecimento de uma radiografia digital sendo determinada pelo $\mathrm{mA}$, e está relacionada aos erros de sub e superexposição. $\mathrm{O} \mathrm{kVp}$ por sua vez está relacionado com o contraste, que torna a imagem final mais visível ${ }^{3}$. A nitidez é descrita por meio do detalhe que sofre influência da distância foco-sensor, distância objeto-receptor e do monitor. Essa unidade determina a capacidade de visualização de detalhes da imagem. A distorção por sua vez está relacionada com fatores como o posicionamento do tubo de raios $\mathrm{X}$ que pode acarretar em alongamento ou encurtamento da imagem final $^{3}$. Um estudo comparou a imagem final analógica, obtida por meio de PSP e pelo sistema CMOS, com o principal objetivo de se observar a qualidade da imagem final. Os autores concluíram que o sistema PSP obteve uma imagem de qualidade satisfatória com uma dose de radiação $70 \%$ menor do que em uma aquisição tradicional, e o CMOS, uma redução de $20 \%{ }^{6}$.

É observado que os sistemas digitais tem uma maior aceitação em relação a latitude de exposição, ou seja, a imagem será considerada de boa qualidade mesmo com uma sub ou superexposição ${ }^{6,7,8}$. Clinicamente, isso implica que se o cirurgião-dentista atentar-se a essa informação, a dose de radiação será diminuída ao paciente. Mas, caso o profissional seja descuidado, a imagem final resultante também será satisfatória mas a dose de radiação será maior ao paciente.

A biossegurança é um aspecto que deve ser observado durante os procedimentos para aquisição das radiografias, especialmente porque os sensores digitais são utilizados por diversos pacientes em vários procedimentos. Todos os posicionadores e receptores de imagem devem ser devidamente protegidos por uma embalagem de PVC e se possível, que o paciente seja atendido em dupla para evitar contaminação da estação de trabalho computadorizada.

Há alguns aspectos legais que destacam também a importância de um sistema de arquivamento digital seguro, com a finalidade de evitar acessos ilegais a base de dados ou mesmo fraudes. As imagens digitais são passíveis de alterações, e, portanto, podem ser usadas para fins de não-diagnóstico que faz com que o cirurgião-dentista armazene com segurança a imagem radiográfica original $^{7}$. Outro problema é o formato de compressão das imagens. Com a finalidade de diminuir o tamanho do arquivo final, a imagem geralmente é salva no formato JPEG. Foi observado que a compressão dos arquivos de imagem do formato TIFF para JPEG pode reduzir em até $96 \%$ o tamanho do arquivo final. No entanto, com essa redução algumas informações são perdidas, como alguns limites anatômicos, e consequentemente, interferir negativamente na realização de medidas por exemplo ${ }^{9}$. 


\section{CONCLUSÃO}

A placa de fósforo representa o sistema digital radiográfico odontológico com maior custo de implatação, mas é o sistema mais confortável para o paciente e mais semelhante, em relação ao posicionamento, às radiografias com filme. O sistema CMOS é mais barato, a visualização da imagem é imediata, e tem como principais desvantagens o desconforto ao paciente e uma imagem final com uma radiopacidade maior que o sistema PSP. Ambos, permitem que a imagem seja editada e armazenada em computadores de modo que facilita o compartilhamento entre os profissionais, e a biossegurança pode ser realizada por meio do uso de embalagem de PVC.

\section{REFERÊNCIAS}

1. Wakoh M, Kuroyanagi K. Digital imaging modalities for dental practice. Bull Tokyo Dent Coll. 2001; 42(1):1-14.

2. Braga EFA, Silva PG, Vardasca de Oliveira PT, Ferrão Júnior JP, Marques J. Comparação das imagens radiográficas digitais e convencionais em reaborções ósseas periodontais. Pesq Bras Odontoped Clin Integr. 2011; 11(4):585-91.

3. Albuquerque AS de; Santos AMS dos; Camelo CMA; Silva GG da; Magalhães TMS; Araújo VGP de; Andrade MEA. Estudo Comparativo entre Sistemas Radiográficos Convencionais e Digitais: Revisão da Literatura. Caderno de Graduação-Ciências Biológicas e da Saúde-FACIPE. 2017; 2(3):99-110.

4. Hehn L, Mahl CRW; Freitas MPM, Conde A, Veeck EB, Fontanella V. Comparação de três sistemas digitais em relação à densidade óptica de cimentos de ionômero de vidro. Rev odonto ciênc. 2007; 22(57): 233-7.

5. Shokri A, Mortazavi H, Salemi F, Javadian A, Bakhtiari $\mathrm{H}$, Matlabi H. Diagnosis of simulated external root resorption using conventional intraoral film radiography, CCD, PSP, and CBCT: a comparison study. Biomed J. 2013; 36(1):18-22.

6. Bhaskaran V, Qualtrough AJ, Rushton VE, Worthington HV, Horner K. A laboratory comparison of three imaging systems for image quality and radiation exposure characteristics. Int Endod J. 2005; 38(9):645-52.

7. Macdonald R. Digital imaging for dentists. Aust Dent J. 2001; 46(4):301-5.

8. Buchanan A, Benton B, Carraway A, Looney S, Kalathingal S. Perception versus reality-findings from a phosphor plate quality assurance study. Oral Surg Oral Med Oral Pathol Oral Radiol. 2017; 123(4):496-501.

9. Yasar F, Apaydin B, Yilmaz HH. The effects of image compression on quantitative measurements of digital panoramic radiographs. Med Oral Patol Oral Cir Bucal. 2012; 17(6):1074-81.

\section{CONFLITO DE INTERESSES}

Os autores declaram não haver conflitos de interesse.

\section{AUTOR PARA CORRESPONDÊNCIA}

Carla Renata Sanomya Ikuta

carla.ikuta@foa.unesp.br

Submetido em 29/03/2018 Aceito em 11/05/2018 